\title{
MULTI-DIMENSIONAL SEPARABLE CRITICALLY SAMPLED WAVELET FILTERBANKS ON ARBITRARY GRAPHS
}

\author{
Sunil K Narang and Antonio Ortega \\ Ming Hsieh Department of Electrical Engineering \\ University of Southern California \\ kumarsun@usc.edu, ortega@sipi.usc.edu
}

\begin{abstract}
In our previous work, we observed an "aliasing" phenomenon for functions defined on bipartite graphs which is analogous to aliasing occurring in the downsampling of regular 1-dimensional signals. We exploited these concepts to design critically sampled two-channel wavelet filterbanks for any bipartite graph. For arbitrary graphs, we proposed a bipartite subgraph decomposition scheme to decompose the graph into edge-disjoint bipartite subgraphs and apply filtering and downsampling separately on each subgraph. This leads to the design of multi-dimensional separable filterbanks on graphs. In this paper, we study these bipartite decompositions in more detail. In particular, we describe the meaning of dimensionality in the subgraph decomposition of arbitrary graphs and define some graph based metrics based on this understanding. Subsequently, we propose a heuristics based algorithm for bipartite subgraph decomposition and compare it with other non-optimized algorithms. The results show both qualitative and quantitative improvements in the decomposed bipartite subgraphs with the proposed heuristics.
\end{abstract}

Index Terms - Nyquist theorem, bipartite graphs, subsampling

\section{INTRODUCTION}

Graphs provide a very flexible model for representing data in many domains such as networks, computer vision, and high dimensional data-clouds. The data on these graphs can be visualized as a finite collection of samples termed as graph-signals. The formulation of datasets as graph-signals has been subject to a lot of study recently. For example, the eigen-vectors $\left\{\mathbf{u}_{l}\right\}_{l=1,2, \ldots N}$ of the undirected graph Laplacian matrix provide a Fourier-like interpretation of graph-signals [1]. The graph Fourier transform (GFT), denoted as $\overline{\mathbf{f}}$, for any signal $\mathbf{f} \in \mathbb{R}^{N}$ on graph $G$ has been defined as $\bar{f}(l)=\left\langle\mathbf{u}_{l}, \mathbf{f}>=\sum_{i=1}^{N} f(i) u_{l}(i)\right.$, where $\bar{f}(l)$ can be interpreted as spectral coefficients of a graph-signal at spectral frequency $\lambda_{l}$. Based on this formulation, Hammond et al [1] defined spectral graph wavelet transforms which are determined by the choice of a continuous bandpass function $g(\lambda)$ in the spectral domain. The multi-channel wavelet transform is constructed from the choice of a low pass kernel $h(\lambda)$ and $J$ band-pass kernels $\left\{g\left(t_{1} \lambda\right), \ldots, g\left(t_{J} \lambda\right)\right\}$. Further, Crovella et al [2] have designed wavelet like functions on graphs which are localized in space and time. Wang et al [3] have proposed graph dependent basis functions for sensor network graphs, which implement an invertible 2-channel like filter-bank. Maggioni and Coifman [4] introduced "diffusion wavelets" as the localized basis functions of the eigenspaces of the dyadic powers of a diffusion operator. A common drawback of all of these filterbank

This work was supported in part by NSF under grant CCF-1018977. designs is that they are not critically sampled. For example, in [1], a $J$-scale decomposition of graph-signal of size $N$ outputs transform coefficients of size $(J+1) \times N$. Therefore the filterbanks require downsampling. Critically sampled lifting based wavelet filterbanks for graphs have been proposed in [5, 6]. However the design requires splitting the vertex set of the graph into two disjoint sets and the links between nodes in the same set are not utilized by the transform. In our previous work in [7], we showed that downsampling in bipartite graphs leads to a spectral folding phenomenon which is analogous to aliasing in regular signal domain. We utilized this property in [8] to propose two channel critically sampled wavelet filterbanks on bipartite graphs. In particular, we design graph-QMF filterbanks, analogous to quadrature mirror filterbanks in the regular signal domain, for any bipartite graphs. These filterbanks cancel aliasing occurring due to downsampling and we define necessary and sufficient conditions for these filterbanks to be perfect-reconstruction and orthogonal. In a general setting, we interpret arbitrary graphs as multi-dimensional bipartite graph and propose an edge-disjoint decomposition of the graph into $K$-bipartite subgraphs. We then apply filtering and downsampling separately to each bipartite subgraph, thus leading to multi-dimensional separable filterbanks.

In this paper we study the desired properties of these bipartite subgraph decompositions. Unlike regular signals, where the filtering and downsampling is done along the geometrical directions (horizontal, vertical etc) of the underlying regular lattice,the graphs do not have a notion of directionality. We describe dimensionality in graphs as separability of neighborhood sets and propose metrics to quantitatively measure the separations. Subsequently, we propose a greedy heuristic to optimize these metrics and compare the resulting decompositions with other non-optimized schemes. The rest of the paper is organized as follows: in Section 2 we describe critically sampled two-channel wavelet filterbanks on arbitrary graphs. The implementation requires a bipartite subgraph decomposition of the given graph. Therefore, in 3 we describe the desired properties of these subgraph decompositions and propose a greedy heuristic to find subgraphs with desired properties. In section 4 we compare proposed algorithm with existing schemes. We conclude the paper in Section 5.

\section{TWO-CHANNEL FILTERBANKS ON GRAPHS}

A two-channel wavelet filterbank on a graph $G$, characterized by filtering operations $\left\{\mathbf{H}_{i}, \mathbf{G}_{i}\right\}_{i=0,1}$ and downsampling operations $\beta_{L}(n)$ and $\beta_{H}(n)$ as shown in Figure 1, provides a decomposition of graph-signal $\mathbf{f}$ into a lowpass (approximation) graph-signal $\mathbf{f}_{\text {low }}$ and a highpass (details) graph-signal component $\mathbf{f}_{\text {high }}$. The transforms $\mathbf{H}_{i}, \mathbf{G}_{i}$ of the two channels are graph transforms containing 


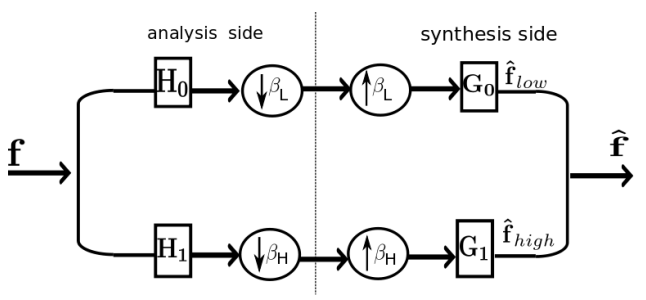

Fig. 1. Block diagram of a two-channel wavelet filterbank on graph.

spatially localized filters. The downsampling function $\beta_{H}$ on the graph $G=(\mathcal{V}, E)$ is defined as choosing a subset $H \subset \mathcal{V}$ such that all samples of the graph signal $\mathbf{f}$, corresponding to indices not in $H$, are discarded, i.e.

$$
\beta_{H}(n)= \begin{cases}1 & \text { if } n \in H \\ -1 & \text { if } n \notin H\end{cases}
$$

A subsequent upsampling operation with $\beta_{H}$ projects the downsampled signal back to original $\mathbb{R}^{N}$ space by inserting zeros in place of discarded samples in $H^{c}$. In matrix form, we define a diagonal downsampling matrix $\mathbf{J}_{\beta_{H}}=\operatorname{diag}\left\{\beta_{H}(n)\right\}$. The overall 'downsample then upsample' $(D U)$ operation can then be algebraically represented as $f_{d u}(n)=1 / 2\left(f(n)+\beta_{H}(n) f(n)\right)$ and in matrix form as $\mathbf{f}_{d u}=1 / 2\left(\mathbf{I}+\mathbf{J}_{\beta_{H}}\right) \mathbf{f}$.

\subsection{Filterbanks on Bipartite Graphs}

In our recent work in $[7,8]$, we observe that for bipartite graphs $G=(L, H, E)^{2}$ choosing the selection set to be either $L$ or $H$ produces a spectral folding phenomenon in the $D U$ signal $\mathbf{f}_{d u}$ which which is analogous to the "aliasing" effect observed in $D U$ operations in regular signal domain. Extending this idea on the twochannel graph filterbank in bipartite graph case, the downsampling functions in Figure 1 are chosen as $\beta=\beta_{L}$ and $\beta_{H}=-\beta$. Thus the nodes in $L$ only retain the output of transform $\mathbf{H}_{0}$ and nodes in $H$ retain the output of transform $\mathbf{H}_{1}$ only and the overall output is critically sampled (i.e., $|L|+|H|=N$ ). The output signals after filtering and $D U$ operation in the lowpass and highpass channels are given as $1 / 2\left(\mathbf{I}+\mathbf{J}_{\beta}\right) \mathbf{H}_{0} \mathbf{f}$ and $1 / 2\left(\mathbf{I}-\mathbf{J}_{\beta}\right) \mathbf{H}_{1} \mathbf{f}$. The overall output $\hat{\mathbf{f}}$ of the filterbank is the sum of outputs of the two channels, i.e., $\hat{\mathbf{f}}=\hat{\mathbf{f}}_{\text {low }}+\hat{\mathbf{f}}_{h i g h}=\mathbf{T f}$, where $\mathbf{T}$ is the overall transfer function of the filterbank given as:

$$
\begin{aligned}
\mathbf{T} & =\frac{1}{2} \mathbf{G}_{0}\left(\mathbf{I}+\mathbf{J}_{\beta}\right) \mathbf{H}_{0}+\frac{1}{2} \mathbf{G}_{1}\left(\mathbf{I}-\mathbf{J}_{\beta}\right) \mathbf{H}_{1} \\
& =\underbrace{\frac{1}{2}\left(\mathbf{G}_{0} \mathbf{H}_{0}+\mathbf{G}_{1} \mathbf{H}_{1}\right)}_{A}+\underbrace{\frac{1}{2}\left(\mathbf{G}_{0} \mathbf{J}_{\beta} \mathbf{H}_{0}-\mathbf{G}_{1} \mathbf{J}_{\beta} \mathbf{H}_{1}\right)}_{B}
\end{aligned}
$$

In (2), $A$ is the transfer function of the filterbank without the $D U$ operation and $B$ arises primarily due to the $D U$ operations. For perfect reconstruction, $\mathbf{T}$ should be equal to identity which means term $A$ should be a scalar multiple of identity and term $B$ should cancel out. In [8], we state necessary and sufficient conditions for a two-channel graph filter-bank on bipartite graphs to provide aliasing-cancellation, perfect reconstruction and orthogonal set of basis (orthogonality).

\footnotetext{
${ }^{2}$ A bipartite graph $G=(L, H, E)$ is a graph whose vertices can be divided into two disjoint sets $L$ and $H$, such that every link connects a vertex in $L$ to one in $H$.
}

Consequently we propose a solution similar to quadrature mirror filters (QMF) in regular signal domain, termed as graph- $Q M F$, which satisfies all of the above conditions.

\subsection{Filterbanks on Arbitrary Graphs}

Not all graphs are bipartite. In order to apply our filterbank design to an arbitrary graph, $G=(\mathcal{V}, E)$, we proposed in [8], a separable downsampling and filtering approach, where our previously designed two-channel filterbanks are applied in a "cascaded" manner, by filtering along a series of $K$ bipartite subgraphs of the original graph. This is illustrated in Figure 3. The bipartite subgraphs cover the same vertex set: $L_{i} \cup H_{i}=\mathcal{V}, i=1,2, \ldots K$, and each edge in $G$ belongs to exactly one bipartite graph. In this approach, a stage of filtering along one "dimension" corresponds to filtering using only those edges that belong to the corresponding bipartite subgraph. Further, in order to guarantee invertibility for structures such as those of Figure 3, given the chosen 2-colorings $\left(H_{i}, L_{i}\right)$, the edge assignment is performed iteratively based on the order of the subgraphs. That is, edges for subgraph 1 are chosen first, then those for subgraph 2 are selected, and so on. An example of decomposition scheme is Harary's algorithm used in [8], which provides a decomposition into $\left\lceil\log _{2} K\right\rceil$ bipartite subgraphs of a $K$-colorable graph. For example, images can be viewed as graph-signals on 4-connected lattice-graph and the links in the image-grid can be decomposed into horizontal and vertical bipartite subgraphs as shown in Figure 2(a). Note that,

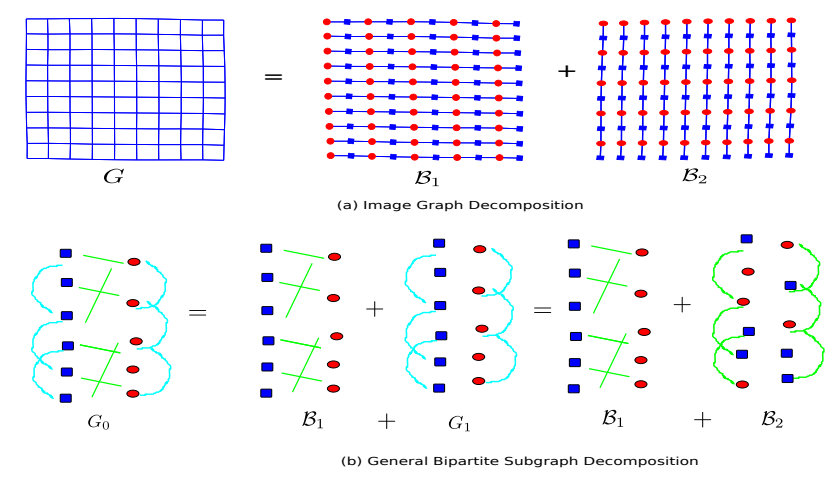

Fig. 2. Bipartite subgraph decomposition scheme.

by construction $G_{1}=G-\mathcal{B}_{1}=\left(\mathcal{V}, E-E_{1}\right)$ contains now two disjoint graphs, since all edges between $L_{1}$ and $H_{1}$ were assigned to $E_{1}$. Thus, at the second stage in Figure $3, \mathcal{B}_{2}$ is composed of two disjoint graphs $\mathcal{B}_{2}\left(L_{1}\right)$ and $\mathcal{B}_{2}\left(H_{1}\right)$, which each will be processed independently by one of the two filterbanks at the second stage. This is also illustrated in Figure 2(b).

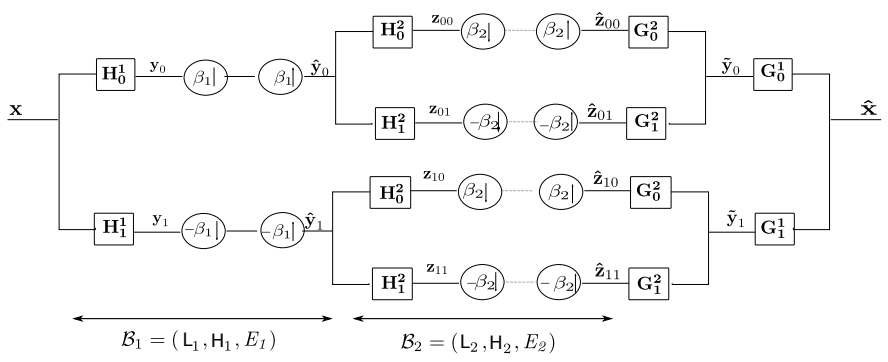

Fig. 3. Separable multi-dimensional filterbank on graphs. 


\section{BIPARTITE SUBGRAPH DECOMPOSITION}

Regular signals possess directionality, for example, a $K$-dimensional regular signal can be visualized as defined on a $k$-dimensional regular lattice-grid which in-turn can be visualized as a Cartesian product of $K$, 1-dimensional lattice-grids. This implies that any point in the lattice can be indexed as $\mathbf{n}=\left(n_{1}, n_{2}, \ldots, n_{K}\right)$ where $n_{k} \in\left\{1,2, . . N_{k}\right\}$. Arbitrary graphs, unlike regular signals, can not be decomposed into Cartesian product of bipartite subgraphs. However like regular signals, arbitrary graphs have $k$-hop neighborhoods $\mathcal{N}_{k}(\mathbf{n})$ at each node $\mathbf{n}$ (in terms of shortest-hop distance). For regular lattice grids these neighborhoods can be decomposed into disjoint neighborhood sets along each direction, i.e., $\mathcal{N}_{k}(\mathbf{n})=\bigcup_{r=1}^{K} \mathcal{N}_{k}\left(n_{r}\right)$. Due to this property the regular signal filters, implemented in each direction (i.e. on the disjoint neighborhoods), filter uncorrelated information about the signal. We extend this property to arbitrary graph and propose a desirable property of bipartite subgraph decompositions $G=\bigcup_{p} \mathcal{B}_{p}$ to be that the $k$-hop neighborhoods $\mathcal{N}_{k}^{p}(n)$ defined on each subgraph $\mathcal{B}_{p}$ are maximally disjoint for all $k$ and for all $n$. The problem is then to find minimum such bipartite subgraph decomposition.

Before finding the solution, we define some metrics which measure the neighborhood separation in bipartite subgraphs. For this, we define $k$-hop adjacency matrix as $\mathbf{A}_{i, k}$ so that $\mathbf{A}_{i, k}(n, m)$ represents the number of paths from node $n$ to node $m$ of length up to $k$. The diagonal entries of $\mathbf{A}_{i, k}$ are set to zero. Using matrix $\mathbf{A}_{i, k}$, we measure separability in the $k$-hop neighborhoods of bipartite subgraphs $\mathcal{B}_{i}$ by computing the correlation between $n^{\text {th }}$ rows of adjacency matrices $\mathbf{A}_{i, k}$ at each node $n$. The $k$-hop neighborhood set correlation NSC $(\mathrm{k})$ between two bipartite subgraphs is the average correlation between the $k$-hop neighborhoods defined as:

$$
N S C(k)=\frac{1}{N} \sum_{n=1}^{N} \frac{\sum_{m} \mathbf{A}_{1, k}(n, m) \mathbf{A}_{2, k}(n, m)}{\sqrt{\sum_{m} \mathbf{A}_{1, k}(n, m)^{2} \sum_{m} \mathbf{A}_{2, k}(n, m)^{2}}}
$$

A low value of NSC would imply mutually disjoint neighborhoods in the decomposed bipartite subgraphs. At a global scale, the eigenvectors of bipartite subgraph Laplacian matrices which form the graph-Fourier basis should also be decorrelated with each other. The correlation between the $l^{\text {th }}$ eigen-vectors $\mathbf{u}_{1, l}$ and $\mathbf{u}_{2, l}$ on two bipartite subgraphs can be measured by their inner-product. Therefore, we define spectral basis correlation SBC between bipartite subgraphs $\mathcal{B}_{1}$ and $\mathcal{B}_{2}$ to be the Euclidean norm of inner-products between the corresponding eigenvectors:

$$
S B C=\sqrt{\sum_{l=1}^{N}\left(\mathbf{u}_{1, l}^{t} \mathbf{u}_{2, l}\right)^{2}}
$$

We next present a heuristic algorithm to find good subgraph decompositions in arbitrary graphs.

\subsection{Min-cut Weighted Max-Cut (MCWMC) algorithm}

The nature and complexity of finding minimum bipartite subgraph decomposition with maximally disjoint neighborhoods is not known. We therefore, propose the following greedy heuristic to find bipartite subgraphs with disjoint neighborhoods: given a graph $G$, let $\beta$ is chosen as the first downsampling function and it induces partition $\left(\mathcal{S}_{1}, \mathcal{S}_{2}\right)$ on graph $G$ with sizes $\left|\mathcal{S}_{1}\right|=N_{1}$ and $\left|\mathcal{S}_{2}\right|=N_{2}$. Further let $\mathbf{e}=E_{\mathcal{S}_{1}, \mathcal{S}_{2}}$ denotes the cut-set and $\mathcal{B}=\left(\mathcal{S}_{1}, \mathcal{S}_{2}, \mathbf{e}\right)$ denotes the bipartite subgraph corresponding to $\beta$. This decomposition can be graphically represented as in Figure 4. The exclusion of $\mathcal{B}$ from $G$ changes the neighborhood structure of the resulting graph $G_{1}$. Thus in remaining graph $G_{1}$, nodes in set $\mathcal{S}_{1}$ can not reach nodes in set $\mathcal{S}_{2}$ and vice-versa. We define the expected change in the neighborhood size at each node given the cut $\mathbf{e}$ as:

$$
E[\partial \mathcal{N} \mid \mathbf{e}]=p\left(\mathcal{S}_{1}\right)\left|\mathcal{S}_{2}\right|+p\left(\mathcal{S}_{2}\right)\left|\mathcal{S}_{1}\right|=\frac{2 N_{1} N_{2}}{N}
$$

Clearly $E[\partial \mathcal{N}]$ is maximized if $N_{1} \approx N_{2}$ at each iteration. This

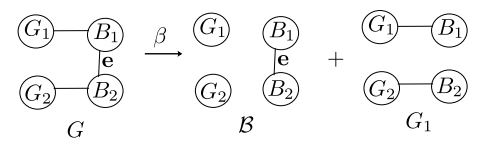

Fig. 4. Example of a bipartite graph-cut

problem is widely studied in graph-literature as the balanced-cut problem in graphs. However finding balanced-cut iteratively becomes problematic as it leads to roughly $\log _{2}(N)$ bipartite subgraphs for graph-size $N$. Further, in each bipartite graphs the nodes which do not have edges in the cut-set e are disjoint and do not take part in the transform. Therefore we would like to maximally pack these edges into larger and fewer bipartite subgraphs, packing edge-sets $\mathbf{e}$ in the order of their importance $E[\partial \mathcal{N} \mid \mathbf{e}]$. In order to do this we assign a weight $w_{e}=E[\partial \mathcal{N}] /|\mathbf{e}|$ to each edge in the cut-set $e \in \mathbf{e}$ in each iteration of balanced cut decomposition. The weight signifies the importance of the edge in changing the neighborhood structure of resulting decompositions. We perform an iterative max-cut algorithm on the resulting min-cut weighted graph which provides bipartite subgraphs with maximum packing of the weighted edges. The algorithm is thus termed as the mincut weighted max-cut (MCWMC) algorithm and illustrated with an example in Figure 5.

\section{EXPERIMENTS}

In order to evaluate the different schemes for bipartite subgraph decomposition, we simulate random graphs by uniformly distributing $N=100$ nodes in a 2-D field and connecting nodes which are within a fixed radius of each other. ${ }^{1}$ For MCWMC algorithm, we use the balanced-cut algorithm proposed in [9] and the max-cut algorithm in [10]. The outcome of proposed algorithm on a sample graph is shown in Figure 5. For each graph $G$, we decompose the graph iteratively into bipartite subgraphs up to two steps to obtain bipartite subgraphs $\mathcal{B}_{1}$ and $\mathcal{B}_{2}$ respectively. To measure the edgepacking in these two subgraphs, we define an edge-loss fraction (ELF) which is the ratio between total number of edges in remaining graph $G_{2}=G-\mathcal{B}_{1}-\mathcal{B}_{2}$ and the total number of edges in $G$. We then evaluate the metrics NSC and SBC for the two bipartite subgraphs obtained by using a) MCWMC algorithm b) Harary's algorithm proposed in [8] and c) a random decomposition (in which we randomly assign downsampling functions to nodes). Table 1 summarizes the comparison results for 100 instances of such random graphs. A low value of ELF suggests that MCWMC algorithm packs more edges in subgraphs $\mathcal{B}_{1}$ and $\mathcal{B}_{2}$ than other algorithms. Further we observe that NSC(k), in general decreases for large $k$-hop neighborhoods which makes sense since at each step of iterative decomposition, the removal of a bipartite subgraph bisects the remaining graph and thus reduces the long-hop connections between nodes.

\footnotetext{
${ }^{1}$ Note that, the 2-D embedding of graph is for illustration only. The MCWMC algorithm only depends on the link-structure of the graph nodes.
} 


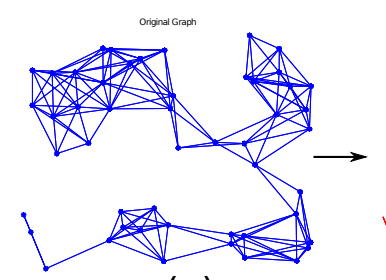

(a)

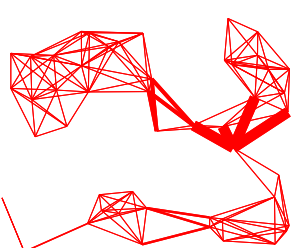

(b)

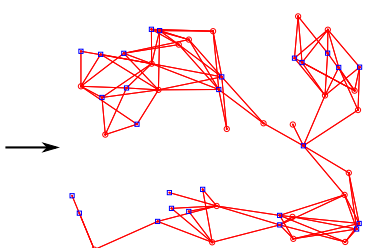

(c)

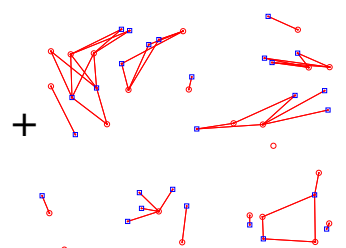

(d)

Fig. 5. Example of MCWMC algorithm steps. (a) original graph (b) converted to a min-cut weighted graph, thickness of an edge represents the weight of the edge. (c) first bipartite subgraph using max-cut (d) second bipartite subgraph.

However we observe that the NSC $(\mathrm{k})$ drops sharply with MCWMC algorithm which implies that the neighborhood are better separated than by using other schemes. At global scale, SBC is lowest for MCWMC algorithm which means that the eigenvectors of the resulting bipartite subgraph are also better decorrelated in case of proposed algorithm. To see it more clearly, we measure similarity (i.e inverse of shortest hoping distance) between all node pairs in different subgraphs. With maximal neighborhood separation, we expect any pair of nodes in the graph to have different similarities on different subgraphs. Figure 6 plots the histogram of absolute difference in the similarities of node-pairs on different bipartite subgraphs. In

\begin{tabular}{|c|c|c|c|}
\hline Method & Random & Harary & MCWMC \\
\hline ELF & 0.249 & 0.225 & 0.14 \\
\hline NSC(2) & 0.48 & 0.53 & 0.51 \\
\hline NSC(4) & 0.50 & 0.54 & 0.51 \\
\hline NSC $(6)$ & 0.49 & 0.53 & 0.48 \\
\hline NSC $(8)$ & 0.47 & 0.51 & 0.45 \\
\hline NSC(10) & 0.45 & 0.49 & 0.42 \\
\hline NSC(12) & 0.43 & 0.48 & 0.39 \\
\hline SBC & 0.60 & 0.61 & 0.55 \\
\hline
\end{tabular}

Table 1. Comparison of bipartite subgraph decomposition schemes

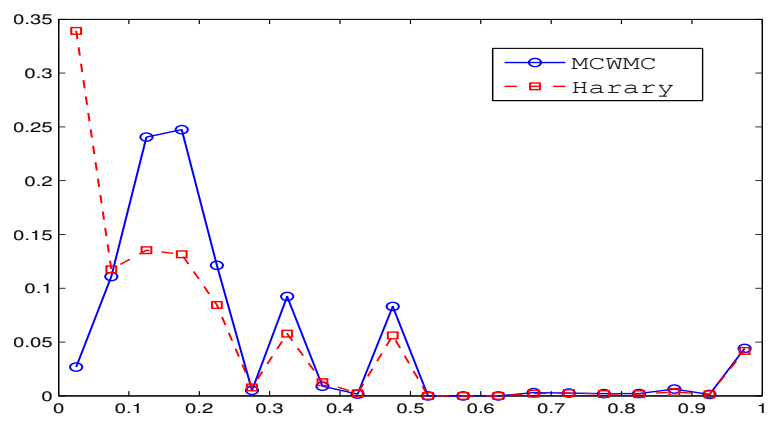

Fig. 6. Histogram of absolute difference in similarity between node-pairs in two bipartite subgraphs

case of Harary's decomposition the histogram is concentrated near zero which means that most node-pairs are near similar on the two subgraphs, whereas in the case of proposed MCWMC algorithm the histogram is shifted to the right implying most node-pairs have different (non-zero) similarities on different bipartite subgraphs. This further corroborates our claim that neighborhood are better separated using the proposed decomposition scheme.

\section{CONCLUSION AND FUTURE WORK}

In this paper, we have explained the design of a critically sampled multi-dimensional filterbanks for arbitrary graphs. According to the original idea proposed in our recent work, a graph is first decomposed into a set of bipartite subgraphs, and filtering and downsampling operations are then carried out in cascade on each bipartite subgraph. In this work, we have proposed bipartite subgraph decompositions which provide dimensionality to the graph, similar to the case of regular signals in higher dimensions, we explained that dimensionality in graphs can be understood as neighborhood separability and we defined some metrics to compare various bipartite decompositions possible for any graph based on this understanding. Further, we defined a heuristics algorithm which improves these metrics. In our future work, we aim to find the efficacy of these graphbased filterbanks vis-à-vis standard wavelet filterbanks on different graph-formulations of the $2 \mathrm{D}$ images.

\section{REFERENCES}

[1] D. K. Hammond, P. Vandergheynst, and R. Gribonval, "Wavelets on graphs via spectral graph theory," CoRR, pp. $-1-1,2009$.

[2] M. Crovella and E. Kolaczyk, "Graph wavelets for spatial traffic analysis," in INFOCOM 2003, Mar 2003, vol. 3, pp. 18481857.

[3] W. Wang and K. Ramchandran, "Random multiresolution representations for arbitrary sensor network graphs," in ICASSP, May 2006, vol. 4, pp. IV-IV.

[4] R. Coifman and M. Maggioni, "Diffusion wavelets," Applied and Computational Harmonic Analysis, vol. 21, pp. 53-94, 2006.

[5] G. Shen and A. Ortega, "Transform-based distributed data gathering," Sig. Proc., IEEE Trans. on, vol. 58, no. 7, pp. 3802 -3815 , july 2010.

[6] S. K. Narang and A. Ortega, "Lifting based wavelet transforms on graphs," (APSIPA ASC' 09), Oct. 2009.

[7] S.K. Narang and A. Ortega, "Downsampling graphs using spectral theory," in ICASSP '11., May 2011.

[8] S.K. Narang and Ortega A., "Perfect reconstruction twochannel wavelet filter-banks for graph structured data," IEEE trans. on Sig. Proc., also avilable in arXiv:1106.3693v3.

[9] Shi J. and Malik J., "Normalized cuts and image segmentation," IEEE Transactions on Pattern Analysis and Machine Intelligence, vol. 22, pp. 888-905, 1997.

[10] B. Aspvall and J. R. Gilbert, "Graph coloring using eigenvalue decomposition,” Tech. Rep., Ithaca, NY, USA, 1983. 